\title{
Development of fast-release piroxicam/polyethylene glycol capsules by solid dispersion and curing using full factorial design
}

\author{
Benchawan Chamsai, Wipada Samprasit* \\ Department of Pharmaceutical Technology, College of Pharmacy, Rangsit University, PathumThani, 12000, Thailand \\ *For correspondence: Email: cbenchawan@gmail.com, swipada@hotmail.com Tel: +66-2997-2222 ext 1404
}

\begin{abstract}
Purpose: To develop fast-release piroxicam (PRX) capsules by solid dispersion with polyethylene glycol (PEG) using melting and curing in a heated coating pan.

Methods: A full factorial design was conducted to investigate the main and interaction effects of molecular weight (4000 - $8000 \mathrm{Da}$ ) and amount (10 - $30 \mathrm{mg}$ PEG to $10 \mathrm{mg}$ PRX) on the dissolution rate of PRX. Temperature $\left(50-70^{\circ} \mathrm{C}\right)$ and duration of the curing process $(15-45 \mathrm{~min})$ were also systematically selected by factorial design.

Results: The molecular weight and amount of PEG significantly impacted on the dissolution rate of $P R X(p=0.04$ and 0.01 , respectively), while temperature and duration of the curing process were not significant effects $(p=0.10$ and 0.17 , respectively). Based on the results, a fast dissolution rate and burst release of PRX was obtained from capsules formulated by PRX/PEG 8000 (1:3 weight ratio) as a solid dispersion compared to the physical mixture and free drug. Furthermore, this capsule was in the acceptance range for the labeled amount, weight variation and disintegration time.

Conclusion: PRX/PEG melted solid dispersion capsule may be a suitable immediate release drug delivery system with improved dissolution rate and increased drug absorption.
\end{abstract}

Keywords: Capsules, Solid dispersion, Piroxicam, Polyethylene glycol, Fast release, Full factorial design

\begin{abstract}
This is an Open Access article that uses a funding model which does not charge readers or their institutions for access and distributed under the terms of the Creative Commons Attribution License (http://creativecommons.org/licenses/by/4.0) and the Budapest Open Access Initiative (http://www.budapestopenaccessinitiative.org/read), which permit unrestricted use, distribution, and reproduction in any medium, provided the original work is properly credited.
\end{abstract}

Tropical Journal of Pharmaceutical Research is indexed by Science Citation Index (SciSearch), Scopus, International Pharmaceutical Abstract, Chemical Abstracts, Embase, Index Copernicus, EBSCO, African Index Medicus, JournalSeek, Journal Citation Reports/Science Edition, Directory of Open Access Journals (DOAJ), African Journal Online, Bioline International, Open-J-Gate and Pharmacy Abstracts

\section{INTRODUCTION}

Solid dispersion involves the dispersion of one or more active ingredients in an inert carrier in the solid state [1] that can increase drug solubility and dissolution by creating a large surface contact area with the dissolution medium. The use of water soluble polymers as carriers optimizes the wetting characteristics of compound surfaces and improves the apparent solubility of drugs. A polymer that has been used extensively as a carrier for solid dispersions is polyethylene glycol (PEG). PEGs with molecular weights of 1500 - $20000 \mathrm{Da}$ are usually employed at various amounts or ratios [2]. Design of experiment (DoE) is a systematic process for optimizing suitable PEGs for solid dispersion, and for determining the relationship 
between PEG and the processing factors affecting dissolution and drug solubility.

A solid dispersion can be prepared by the melting, solvent, or melting-solvent methods [3]. The melting method is an approach that drug is dispersed in a melted carrier, after that the mixture is rapidly solidified. However, the solid dispersion products need to be further prepared into dosage forms including tablets and capsules that requires several processes. In the case of capsules, solid dispersion and excipients are filled into hard gelatin capsules, indicating more processing steps are required, which is difficult to scale up for commercial production. Another way to minimize the processes is by preparing physical mixture of the drug, carrier and excipient, filled into hard gelatin capsules and then cured in a temperature controlled machine. However, the effects of temperature and curing duration time need to be optimized to achieve the best dissolution rate of the drug.

Piroxicam (PRX) is a nonsteroidal antiinflammatory drug with low water solubility. PRX is absorbed slowly and gradually through the gastrointestinal tract and, consequently, the onset of analgesic and anti-inflammatory actions is delayed [4]. By improving the drug release profile of PRX, it is possible to enhance its bioavailability and therapeutic actions [5]. Therefore, capsule formulations of fast releasing PRX using solid dispersion with PEG are interesting and may increase the solubility of PRX. The aim of this study was to develop fast releasing PRX/PEG capsules by melted solid dispersion and curing in heated coating pan. $\mathrm{A}^{2}$ full factorial design was used to study the effect of molecular weight and amount of PEG on the dissolution rate of PRX capsules prepared by melted solid dispersion. Processing factors including temperature and time of curing on the physical mixtures of PRX and PEG capsules were also studied using this design. Prepared capsules were evaluated for PRX labeled amount, weight variation, disintegration time, dissolution profile and rate compared to the physical mixtures and free drug capsules.

\section{EXPERIMENTAL}

\section{Materials}

PRX was received from T.O. Co., Ltd, Thailand. PEG 4000, 6000 and 8000, Lactose and colloidal silicon dioxide $\left(\right.$ Aerosil ${ }^{\circledR}$ ) were obtained from Sigma Chemical Co., USA. Hard gelatin capsule of size 1 was purchased from Thai Herbal Group (Bangkok, Thailand). All other reagents and solvents were analytical grade. The deionized water was used in this research study.

\section{Preparation of PRX/PEG melted solid dispersion capsules}

PEG was accurately weighed, placed in an oil bath and stirred continuously until it completely melted. The temperature of the oil bath was set at $80{ }^{\circ} \mathrm{C}$ and the actual temperature of melted PEG was the interval of $65-70^{\circ} \mathrm{C}$. Then, PRX was placed into the melted PEG and mixed continuously until all materials were homogenous. The mixture was immediately solidified in an ice bath. The solid dispersion obtained was ground, passed through a sieve size 60 (opening size $0.250 \mathrm{~mm}$ ) and stored in a desiccator before filling into the capsules.

The preparation was prepared and designed by a $3^{2}$ full factorial design. The software DESIGNEXPERT $^{\circledR}$ version 10 (Trial version, Stat-Ease Inc., Minneapolis, USA) was used to preformed the Statistical experimental design. Response surface graphics were used to study factor interaction between the considered variables. Selected independent variables were the average molecular weight of PEG $\left(X_{1}\right)$ and the amount of PEG $\left(X_{2}\right)$. The factorial levels were coded as low, medium, and high settings $(+1,0$ and -1 , respectively) for the independent variable. The average molecular weights of PEG $\left(X_{1}\right)$ were 4000,6000 and 8000 , and amounts of PEG $\left(X_{2}\right)$ were 10,20 and $30 \mathrm{mg} /$ capsule. Different amounts of PEG at 10, 20 and 30 $\mathrm{mg} / \mathrm{capsule}$ were selected to represent PRX:PEG weight ratios at 1:1, 1:2 and 1:3, respectively. These ratios were used to assess the effect of increasing PEG concentration on the dissolution rate of PRX. The selected response investigated was the dissolution rate of $P R X$ from capsules at $3 \mathrm{~min}(\mathrm{Y})$. The number of trials required for the study was based on the number of independent variables selected [6]. The nine experimental runs were required for analyzing the interaction of each level. Table 1 shows the factors with their levels. Table 2 shows the responses of the variables. The response $(Y)$ in each trial was measured by conducting a multiple linear regression (MLR) using the factorial model in Eq 1.

$Y=b_{0}+b_{1} X_{1}+b_{2} X_{2}+b_{12} X_{1} X_{2}$

where $Y$ is the response, $b_{0}$ is the arithmetic mean response of all trials, and $b_{i}$ is the estimated coefficient for factor $X_{i}$. The main effects, $X_{1}$ and $X_{2}$, represent the average value of changing factors one at a time, and $X_{1} X_{2}$ represents the interaction terms. 
PRX/PEG solid dispersion capsules were developed containing an equivalent dose $(10 \mathrm{mg})$ of PRX formed by solid dispersion, physical mixture and free drug. Compositions are presented in Table 3. Lactose was selected as a filler due to it is highly water soluble which commonly used in the capsules. Aerosil ${ }^{\circledR}$ was used as a glidant to optimize the flow of powders into the capsules. Batch production size was 150 capsules. The solid dispersion and lactose were mixed in a plastic bag for $10 \mathrm{~min}$. Then, the glidant was added and mixed further for $5 \mathrm{~min}$. Powders were filled into hard gelatin capsules of size 1 in a lab scale capsule filling machine (Semiautomatic Capsule Filling Machine (Model Panviv A-01)).

\section{Preparation of PRX/PEG capsules by curing in a heated coating pan}

PRX and PEG 8000 at a weight ratio of $1: 3$ were selected to prepare the capsules by curing in a heated coating pan (Hyealhang Co. Ltd., Bangkok, Thailand) at a speed of $60 \mathrm{rpm}$. PRX was physically mixed with PEG 8000 in a plastic bag for $10 \mathrm{~min}$. Then, lactose was added and mixed further for $10 \mathrm{~min}$. Finally, the additional Aerosil $^{\circledR}$ was mixed for 5 min. Powders were filled into hard gelatin capsules of size 1 in a lab scale capsule filling machine. The prepared capsules were cured in a heated coating pan at various temperatures $\left(50,60\right.$ and $70{ }^{\circ} \mathrm{C}$ ) and times (15, 30 and $45 \mathrm{~min}$ ).

Table 1: Variables and their levels for $3^{2}$ full factorial design

\begin{tabular}{|c|c|c|c|c|}
\hline \multirow[t]{2}{*}{$\begin{array}{l}\text { Independent } \\
\text { variables }\end{array}$} & \multicolumn{2}{|c|}{$\begin{array}{l}\text { PRX/PEG melted solid dispersion } \\
\text { capsules }\end{array}$} & \multicolumn{2}{|c|}{$\begin{array}{l}\text { PRX/PEG capsules curing in the } \\
\text { heated coating pan }\end{array}$} \\
\hline & \multicolumn{4}{|c|}{ Actual values } \\
\hline Coded value & $\begin{array}{c}\text { Average } \\
\text { molecular weight } \\
\text { of PEG }\left(X_{1}\right)\end{array}$ & $\begin{array}{c}\text { Amount of PEG } \\
\text { (mg) }\left(X_{2}\right)\end{array}$ & $\begin{array}{c}\text { Temperature }\left({ }^{\circ} \mathrm{C}\right) \\
\left(X_{1}\right)\end{array}$ & Time $(\min )\left(X_{2}\right)$ \\
\hline Low $(-1)$ & 4000 & 10 & 50 & 15 \\
\hline Medium (0) & 6000 & 20 & 60 & 30 \\
\hline High $(+1)$ & 8000 & 30 & 70 & 45 \\
\hline $\begin{array}{l}\text { Dependent va } \\
\text { Dissolution rat }\end{array}$ & m capsules & $\operatorname{lin}(Y)$ & & \\
\hline
\end{tabular}

Table 2: The $3^{2}$ full factorial design and responses of PRX/PEG capsules. Data are expressed as mean \pm SD $(n$ =6)

\begin{tabular}{|c|c|c|c|c|c|c|c|}
\hline \multicolumn{4}{|c|}{ PRX/PEG melted solid dispersion capsules } & \multicolumn{4}{|c|}{$\begin{array}{l}\text { PRX/PEG capsules curing in the heated coating } \\
\text { pan }\end{array}$} \\
\hline \multirow{2}{*}{ Batch } & \multicolumn{2}{|c|}{$\begin{array}{l}\text { Independent variables } \\
\text { in coded value }\end{array}$} & \multirow{2}{*}{$\begin{array}{l}\text { Dependent } \\
\text { variables Y }\end{array}$} & \multirow{2}{*}{ Batch } & \multicolumn{2}{|c|}{$\begin{array}{l}\text { Independent variables } \\
\text { in coded value }\end{array}$} & \multirow{2}{*}{$\begin{array}{l}\text { Dependent } \\
\text { variables Y }\end{array}$} \\
\hline & $\mathrm{X}_{1}$ & $X_{2}$ & & & $\mathrm{X}_{1}$ & $\mathrm{X}_{2}$ & \\
\hline $\mathrm{F} 1$ & -1 & -1 & $4.18 \pm 0.34$ & $\mathrm{~F} 11$ & -1 & -1 & $5.47 \pm 0.70$ \\
\hline $\mathrm{F} 2$ & 0 & -1 & $3.86 \pm 0.01$ & $\mathrm{~F} 12$ & 0 & -1 & $5.42 \pm 0.72$ \\
\hline F3 & 1 & -1 & $4.89 \pm 0.01$ & F13 & 1 & -1 & $6.11 \pm 5.32$ \\
\hline $\mathrm{F} 4$ & -1 & 0 & $4.75 \pm 0.29$ & F14 & -1 & 0 & $5.50 \pm 0.71$ \\
\hline F5 & 0 & 0 & $5.02 \pm 0.70$ & F15 & 0 & 0 & $5.41 \pm 0.85$ \\
\hline F6 & 1 & 0 & $5.46 \pm 0.01$ & F16 & 1 & 0 & $5.83 \pm 0.74$ \\
\hline F7 & -1 & 1 & $4.89 \pm 0.54$ & F17 & -1 & 1 & $5.41 \pm 0.68$ \\
\hline F8 & 0 & 1 & $5.49 \pm 0.47$ & F18 & 0 & 1 & $5.53 \pm 0.43$ \\
\hline F9 & 1 & 1 & $5.56 \pm 0.16$ & F19 & 1 & 1 & $5.39 \pm 0.55$ \\
\hline
\end{tabular}

Table 3: Composition of capsules containing the PRX formed in solid dispersion, physical mixture and free drug

\begin{tabular}{|c|c|c|c|c|c|c|c|c|c|c|c|}
\hline $\begin{array}{l}\text { Composition } \\
\text { (mg) }\end{array}$ & Fo & $\mathbf{F 1}$ & $\mathbf{F 2}$ & F3 & F4 & F5 & F6 & F7 & F8 & F9 & F10 \\
\hline PRX & $10^{*}$ & 10 & 10 & 10 & 10 & 10 & 10 & 10 & 10 & 10 & $10^{\star *}$ \\
\hline PEG 4000 & - & 10 & - & - & 20 & - & - & 30 & - & - & - \\
\hline PEG 6000 & - & - & 10 & - & - & 20 & - & - & 30 & - & - \\
\hline PEG 8000 & - & - & - & 10 & - & - & 20 & - & - & 30 & 30 \\
\hline Aerosil $^{(\mathbb{B})}$ & 6 & 6 & 6 & 6 & 6 & 6 & 6 & 6 & 6 & 6 & 6 \\
\hline Lactose & 288.4 & 278.4 & 277.3 & 277.3 & 268.4 & 267.3 & 267.3 & 258.4 & 257.3 & 257.3 & 257.3 \\
\hline
\end{tabular}


The coating pan was cured at each temperature for at least $30 \mathrm{~min}$ before the curing process to ensure that the actual temperature was equal to that of the coating pan setting temperature. The effect of thermal processes (temperature and time) on the dissolution rate of PRX from capsules at $3 \mathrm{~min}$ was systematically studied using software DESIGN-EXPERT ${ }^{\circledR}$ by a three level, two factor full factorial design. The three factorial levels of independent factors (temperature $\left(X_{1}\right)$ and the time $\left(X_{2}\right)$ ), and dependent factor (dissolution rate of PRX from capsules at $3 \min (\mathrm{Y})$ ) are presented in Table 1. Table 2 shows the responses of variables $(Y)$, measured by carrying out an MLR using the factorial model as Eq 1.

\section{Evaluation of PRX/PEG capsules}

\section{PRX content}

The amount of PRX in a capsule was determined by dissolving $P R X$ in simulated gastric fluid without pepsin which consisted of $2 \mathrm{~g} \mathrm{NaCl}$ and 7 $\mathrm{ml}$ conc. $\mathrm{HCl}$ per liter of deionized water, adjusted to $\mathrm{pH} 1.2 \pm 0.01$ [7]. Ten capsules were taken randomly, opened and poured into a mortar. The crushed powder was weighed and transferred to a $100 \mathrm{ml}$ volumetric flask, then 50 $\mathrm{ml}$ of methanol and $50 \mathrm{ml}$ of simulated gastric fluid without pepsin were added to adjust the volume. The mixture was stirred on a magnetic stirrer, diluted by simulated gastric fluid without pepsin and assayed using a UV-visible spectrophotometer (Spectronic ${ }^{\circledR}$ Genesyn 5, Rochester, U.S.A.) at a wavelength of $333 \mathrm{~nm}$. The PRX content was calculated and expressed as labeled amount (\%) using Eq 2.

Labeled amount $(\%)=\frac{\mathrm{PRX}_{\mathrm{a}}}{\mathrm{PRX}_{\mathrm{T}}} \times 100 \ldots$

where $P R X_{a}$ is the amount of PRX analyzed in the capsules and $P R X_{t}$ is the labeled amount of PRX $(10 \mathrm{mg})$ in the capsules.

\section{Weight variation}

Twenty capsules were individually weighed on an analytical balance (Sartorius AG Gottingen. Model AC 2115, Germany). The average weight and standard deviation were calculated.

\section{In vitro disintegration time}

Disintegration of capsules in deionized water was determined using a USP disintegration testing apparatus (K.S.L. Engineering Company, Thailand) [7]. The medium was maintained at 37 $\pm 0.5^{\circ} \mathrm{C}$ throughout the test. Six capsules were placed into a basket-rack assembly and disintegration time was recorded when the capsules disintegrated and passed through the screen of the basket-rack assembly.

\section{In vitro dissolution}

USP dissolution testing apparatus I (Pharma test Apparatebau GmbH. Model TW II Hainburg, Germany) was used to determine PRX release from the capsules $(n=6)$. The study examined simulated gastric fluid without pepsin $(900 \mathrm{ml})$ maintained at $37 \pm 0.5{ }^{\circ} \mathrm{C}$ with a rotation at 50 $\mathrm{rpm}$. The samples $(10 \mathrm{ml})$ were withdrawn from dissolution vessels at $3,6,9,12,15,18,25,35$ and $45 \mathrm{~min}$, and replaced with fresh medium. The amount of released PRX was analyzed by UV at $333 \mathrm{~nm}$. Dissolution rate of PRX at $3 \mathrm{~min}$ (D3) was calculated for the various formulations as in Eq 3.

$\mathrm{D} 3(\mathrm{~W} / \mathrm{min})=\frac{\mathrm{FRX}_{\mathrm{at}} 3 \min }{3}, \ldots \ldots \ldots \ldots \ldots(3)$

where, $P R X_{a t} 3$ min is the percentage of $P R X$ release at $3 \mathrm{~min}$.

\section{Statistical analysis}

Evaluated capsule data were expressed as means \pm standard deviation (SD) and various response surface methodology computations and mathematic equations were assessed for reliability. Three formulations were randomly selected to estimate the dissolution rate using the generated mathematical models from the experimental procedures. Observed (Ob) and predicted dissolution rate $(\operatorname{Pr})$ values were compared, and the percent error calculated in prognosis (\%PE) using Eq 4.

$\mathrm{PE}(\%)=\{(\mathrm{Ob}-\mathrm{Pr}) / \mathrm{Ob}\}$

The difference in dissolution rate of PRX from the selected capsules was analyzed by using oneway analyses of variance (ANOVA). The significance level was set to $p<0.05$.

\section{RESULTS}

\section{PRX/PEG melted solid dispersion capsules}

Homogeneous mixtures occurred along the process and the yellowish powder of solid dispersion was observed by visual inspection. PRX/PEG solid dispersions capsules were no significant differences in the labeled amount and weight of these capsules (Table 4). For all capsules, the percentage of labeled amount was within $92.5-107.5 \%$ which was within the criteria 
of pharmacopoeia [7] and the weight was $\approx 300$ $\mathrm{mg} /$ capsule. Capsules rapidly disintegrated in 2-3 min, an acceptable limit for capsules (less than 15 min). PRX dissolution profiles of capsules are shown in Figure 1 A. PRX formed in the various solid dispersion capsules provided the different amounts and rates of dissolution within $15 \mathrm{~min}$ of the dissolution study. After that, PRX released slowly from the capsules for 45 min. However, all capsules released PRX at not less than $75 \%$ of the labeled amount in 45 min, which was within the criteria of pharmacopoeia [7].
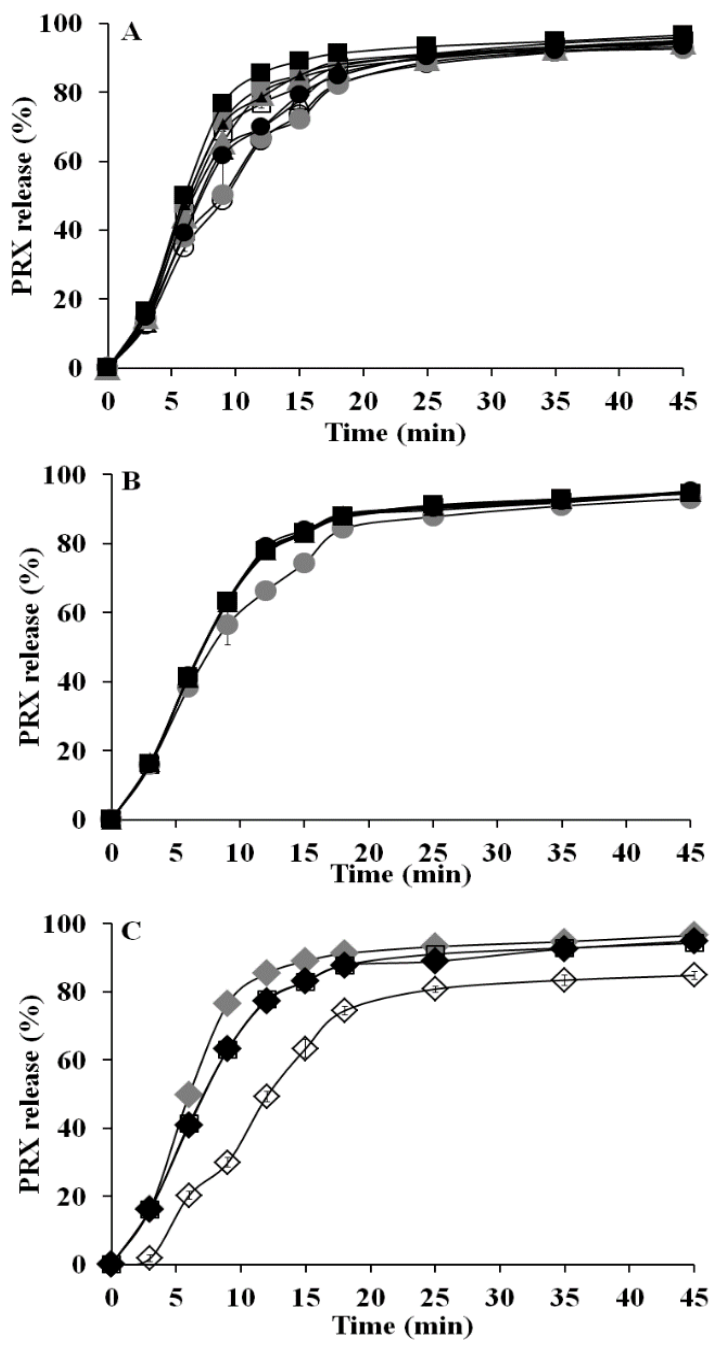

Figure 1: The PRX release profiles from (A) PRX/PEG melted solid dispersion capsules; ( $(\circ) \mathrm{F1},(\Delta) \mathrm{F} 2(\square) \mathrm{F} 3$,

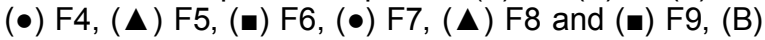
$\mathrm{PRX} / \mathrm{PEG}$ capsules curing in the heated coating pan

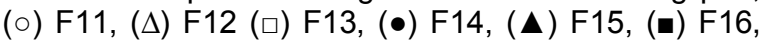
$(\bullet) F 17,(\Delta) F 18$ and (a) F19 and (C) the selected

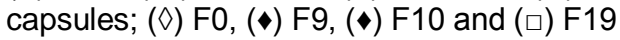

The different average molecular weights $\left(X_{1}\right)$ and amount $\left(X_{2}\right)$ of PEG showed change in dissolution rate of $P R X$ at 3 min (Y) (Table 2). It was found that for responses $Y$, linear contribution models were significant $(p=0.0266)$ with $R^{2}$ at 0.8186 . Table 5 shows the factor effects and associated p-values of each response factor. Factors $X_{1}$ and $X_{2}$ gave significant $p$-values, while interaction between the considered variables $\left(X_{1} X_{2}\right)$ was not significant (Table 5). Positive values were observed by factors $X_{1}$ and $X_{2}$, while interaction of $X_{1} X_{2}$ was negative. Moreover, a higher factor effect of $X_{2}$ than $X_{1}$ and $X_{1} X_{2}$.

Table 5: The quantitative factor effects and associated $P$ value for the response

\begin{tabular}{|c|c|c|c|c|}
\hline \multirow[b]{3}{*}{ Factor } & \multicolumn{2}{|c|}{$\begin{array}{c}\text { PRX/PEG melted } \\
\text { solid dispersion } \\
\text { capsules }\end{array}$} & \multicolumn{2}{|c|}{$\begin{array}{c}\text { PRX/PEG capsules } \\
\text { curing in the heated } \\
\text { coating pan }\end{array}$} \\
\hline & \multicolumn{2}{|c|}{$\mathbf{Y}$} & \multicolumn{2}{|c|}{$\mathbf{Y}$} \\
\hline & $\begin{array}{l}\text { Factor } \\
\text { effect }\end{array}$ & P-value & $\begin{array}{l}\begin{array}{l}\text { Factor } \\
\text { effect }\end{array} \\
\end{array}$ & P-value \\
\hline$X_{1}$ & 0.35 & 0.04232 & 0.1405 & 0.1048 \\
\hline$X_{2}$ & 0.5 & 0.01139 & $0 . \overline{-}$ & 0.1714 \\
\hline$X_{1} X_{2}$ & -0.01 & 0.95183 & $0 . \overline{-}$ & 0.1172 \\
\hline
\end{tabular}

Figure 2 shows the 2-D contour and 3-D response surface plots of the effect of $X_{1} X_{2}$ on the response $Y$. The observed value for $Y$ ranged from 3.86 to $5.56 \% / \mathrm{min}$. Once the decoded values of factor levels were applied and a response was performed, linear regression was obtained in terms of coded factors as Eq 5 . The equation in terms of actual factors was also performed and described as Eq 6:

$Y=4.90+0.35 \mathrm{X}_{1}+0.50 \mathrm{X}_{2}-0.01 \mathrm{X}_{1} \mathrm{X}_{2}$

$$
\mathrm{D} 3=2.79+0.00018 \mathrm{MW}+0.05317 \mathrm{~A}
$$$$
-0.0000005 \text { (MW)(A) }
$$

where D3 is the dissolution rate at $3 \mathrm{~min}$, MW and $A$ are the molecular weight and amount in milligram of PEG, respectively.

The observed and predicted values of the response and \%PE are shown in Table 6. There was not much difference between observed and predicted value. The \%PE was about 1 to $3 \%$.
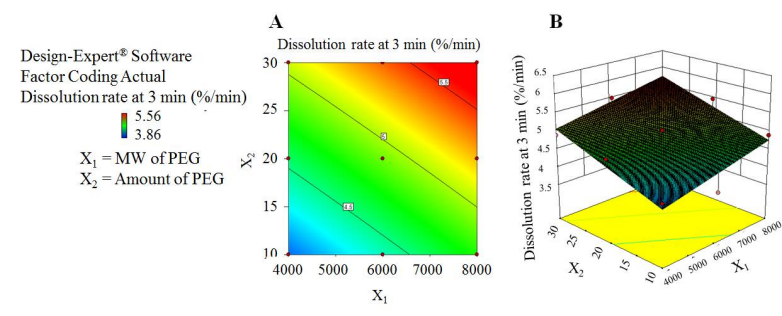

Figure 2: $(A)$ Contour plot and $(B)$ response surface plot for the dissolution rate at $3 \mathrm{~min}$ of $P R X / P E G$ melted solid dispersion capsules

Trop J Pharm Res, June 2018; 17(6): 985 
Table 4: Characteristics of capsules containing the PRX formed in solid dispersion, physical mixture and free drug. Data are expressed as mean \pm SD $(n=6)$

\begin{tabular}{|c|c|c|c|c|c|c|c|c|c|c|c|}
\hline Variable & Fo & $\mathbf{F 1}$ & $\mathbf{F 2}$ & F3 & F4 & F5 & F6 & $\mathbf{F 7}$ & F8 & F9 & F10 \\
\hline Labeled amount (\%) & 96.34 & 92.95 & 96.34 & 99.05 & 96.34 & 97.60 & 98.37 & 96.34 & 98.37 & 99.73 & 99.73 \\
\hline Weight variation (mg) & $302.4 \pm 12.1$ & $301.4 \pm 8.1$ & $302.4 \pm 12.1$ & $298.8 \pm 7.0$ & $300.1 \pm 9.3$ & $297.4 \pm 7.1$ & $298.2 \pm 6.0$ & $301.6 \pm 9.9$ & $300.2 \pm 6.8$ & $302.3 \pm 5.5$ & $301.1 \pm 10.2$ \\
\hline Dissolution rate at $3 \mathrm{~min}(\% / \mathrm{min})$ & $0.61 \pm 0.32$ & $4.18 \pm 0.34$ & $3.86 \pm 0.01$ & $4.89 \pm 0.01$ & $4.75 \pm 0.29$ & $5.02 \pm 0.70$ & $5.46 \pm 0.01$ & $4.89 \pm 0.54$ & $5.49 \pm 0.47$ & $5.56 \pm 0.16$ & $5.41 \pm 0.39$ \\
\hline
\end{tabular}


Table 6: Observed and predicted values (the dissolution rate at $3 \mathrm{~min}$ ) of the selected PRX/PEG melted solid dispersion capsules and the percentage prognosis error

\begin{tabular}{lccccc}
\hline \multirow{2}{*}{$\begin{array}{l}\text { Independent variables in } \\
\text { coded value }\left(\mathrm{X}_{\mathbf{1}}: \mathbf{X}_{\mathbf{2}}\right)\end{array}$} & $\begin{array}{c}\text { Observed } \\
\text { value }\end{array}$ & \multicolumn{2}{c}{$\begin{array}{c}\text { Equation in term of } \\
\text { coded factors }\end{array}$} & \multicolumn{2}{c}{$\begin{array}{c}\text { Equation in term of } \\
\text { actual factors }\end{array}$} \\
\cline { 3 - 6 } & & $\begin{array}{c}\text { Predicted } \\
\text { value }\end{array}$ & $\% P E$ & $\begin{array}{c}\text { Predicted } \\
\text { value }\end{array}$ & $\% P E$ \\
\hline$-1:-1$ & 4.18 & 4.04 & 3.35 & 4.02 & 3.79 \\
$0:+1$ & 5.49 & 5.40 & 1.64 & 5.38 & 2.09 \\
$+1:-1$ & 4.89 & 4.76 & 2.66 & 4.72 & 3.44 \\
\hline
\end{tabular}

Table 7: Dissolution rate at pre-set time-points for the selected capsules

\begin{tabular}{lcccc}
\hline Time-point & F0 & F9 & F10 & F19 \\
\hline 3 min & $0.61 \pm 0.32$ & $5.56 \pm 0.16$ & $5.41 \pm 0.39$ & $5.39 \pm 0.55$ \\
6 min & $3.38 \pm 0.18$ & $8.31 \pm 0.32$ & $6.79 \pm 0.22$ & $6.89 \pm 0.24$ \\
$9 \min$ & $3.33 \pm 0.15$ & $8.52 \pm 0.24$ & $7.02 \pm 0.10$ & $7.01 \pm 0.19$ \\
$15 \mathrm{~min}$ & $4.22 \pm 0.18$ & $5.54 \pm 0.15$ & $5.54 \pm 0.15$ & $5.53 \pm 0.19$ \\
$25 \mathrm{~min}$ & $3.23 \pm 0.04$ & $3.56 \pm 0.04$ & $3.56 \pm 0.04$ & $3.54 \pm 0.04$ \\
\hline
\end{tabular}

\section{PRX/PEG capsules by curing in a heated coating pan}

PRX, PEG 8000 and other excipients were mixed, filled into capsules as PRX/PEG 8000 physical mixture capsules and then heated at various temperatures and times. Figure $1 \mathrm{~B}$ presents PRX dissolution profiles from PRX/PEG capsules by curing in the heated coating pan. There were no differences in the dissolution profiles from the various capsules. All capsules released $\mathrm{PRX}$ at similar dissolution rates (Table 2) within $45 \mathrm{~min}$ of testing. Capsules released PRX at not less than $75 \%$ of the labeled amount in $45 \mathrm{~min}$, which was within the criteria of pharmacopoeia [7].

Table 1 and Table 2 show the factors and the response of the design, respectively. A similar dissolution rate of $\mathrm{PRX}$ at $3 \min (\mathrm{Y})$ was observed by changing the temperature $\left(X_{1}\right)$ and time $\left(X_{2}\right)$ of the curing process. Insignificant MLR model $(p=0.0954)$ was considered with $\mathrm{R}^{2}$ of 0.6910 . Furthermore, the factor effects for the response were also not significant (Table 5). The $p$-value obtained by temperature $\left(X_{1}\right)$, time $\left(X_{2}\right)$ and the interaction of temperature and time $\left(\mathrm{X}_{1} \mathrm{X}_{2}\right)$ was more than 0.05 .

\section{Selection of optimized PRX/PEG capsules}

The characteristics of selected capsules are illustrated in Table 4. The PRX labeled amount, weight variation and in vitro disintegration time were not significantly different between these formulations. However, in vitro dissolution studies showed different dissolution patterns (Figure $1 \mathrm{C}$ ). Capsules containing the free drug (F0) slowly released $\sim 60 \%$ of the PRX within 45 min, which was less than the other formulae (F9, $\mathrm{F} 10$ and $\mathrm{F} 19)$. Also, the dissolution rate of $\mathrm{PRX}$ from free drug capsules was very low at several times (Table 7).

Capsules containing PRX/PEG 8000 melted solid dispersion (F9), physical mixture (F10) and cured physical mixture (F19) completely released 100 $\%$ of the MX within $45 \mathrm{~min}$, which was more than the free drug (F0). However, capsules containing PRX/PEG 8000 melted solid dispersion (F9) gave a faster release of PRX than the physical mixture with and without curing process (F19 and F10, respectively). F9 had a significantly higher dissolution rate at 3, 6 and 9 min than the other formulae (Table 7). A similar dissolution profile (Fig. $2 \mathrm{C}$ ) and rate (Table 7) of capsules containing the physical mixture with and without curing process (F19 and F10, respectively) was found. Thus, the order of PRX dissolution rate enhancement was $\mathrm{F} 9>\mathrm{F} 19 \approx \mathrm{F} 10>\mathrm{F} 0$. Dissolution rate at 3,6 and 9 min of PRX from melted solid dispersion capsules was 12, 2.5 and 2.5 fold higher than the free drug capsules, respectively.

\section{DISCUSSION}

Various PRX/PEG solid dispersions were successfully prepared by the melting method. It was likely that PRX was dispersed within the matrix of PEG. The structure of PRX contains the carbonyl $(\mathrm{C}=\mathrm{O}), \mathrm{N}-\mathrm{H}$ and $\mathrm{O}-\mathrm{H}$ groups that may form hydrogen bonds with a number of $\mathrm{O}-\mathrm{H}$ groups of PEG [8]. Rapid disintegration of the capsules was due to the hydrophilic surface of solid dispersion. The hydrophilic nature of PEG was attributed to the presence of water and absorbed moisture from the environment [9], which accelerated capsule disintegration. Capsules formulated with high contents of PEG showed faster disintegration than those with lower amounts. 
Different dissolution rates of PRX from capsules were caused by the solid dispersion of PRX loaded into the capsules. The PEG molecular weight appeared to have an impact on the solid dispersion composition which exhibited specific interactions with PRX [10]. The amount of polymer or drug/polymer ratio also affected the free drug concentration and drug solubility [11]. Increase in percentage and dissolution rate of PRX release from the capsules with increase of molecular weight and amount of PEG was due to the concomitant increase in hydrophilic surface and wettability of $P R X$, as well as the increased polymer chain and amount of PEG, resulting in enhanced diffusion of the dissolution medium and the release of PRX.

A $3^{2}$ full factorial design was used to investigate the effect of average molecular weight $\left(X_{1}\right)$ and amount of PEG $\left(X_{2}\right)$ on PRX release from the capsules. The $X_{1}$ and $X_{2}$ positively affected the dissolution rate of PRX. The increasing in PEG molecular weight with hydrophilic surface increased the dissolution rate of PRX. In all examined cases of factor $X_{2}$ with different amounts of $P E G$, an increase in weight fraction of PEG resulted in an improvement in the PRX dissolution rate. Possible reasons include the facilitation PRX dissolution by dissolving PEG and a decrease in the particle size of PRX in the PEG carrier, with an increase in PEG concentration [12]. The amount of PEG was dominant for the dissolution rate of PRX over the molecular weight of PEG.

The 2-D contour and 3-D response surface plots are the graphical representation of the regression equation used to visualize the relationship between the response and experimental levels of each factor. The Eq 5 and 6 can be used to make predictions about the response for given levels of each factor. The reliability of equations was assessed by comparing the observed and predicted values from these equations. The less $\% \mathrm{PE}$ values indicated reliability of the optimization equation and procedure. These results corresponded with $R^{2}$, indicating a good prediction of the equation.

Based on the linear regression equation, the optimized capsule produced from PRX/PEG 8000 solid dispersion at weight ratio of 1:3 PRX to PEG 8000 (F9) provided the highest dissolution rate at $3 \mathrm{~min}$. Thus, this capsule formulation was selected to compare the characteristics with PRX/PEG capsules by curing in a heated coating pan at the same molecular weight and amount of PEG, free drug capsules and physical mixture capsules.
This technique has one processing step, compared to melted solid dispersion. The capsules could be prepared without the cooling down, pulverization and sieving processes. This method also avoided producing materials with soft, tacky and poor flow properties [13]. During the curing process, capsules were rotated in the coating pan with thermal processes, providing dispersed PRX in melted PEG 8000, while lactose and Aerosil ${ }^{\circledR}$ were mixed in PRX/PEG 8000 mixture [14]. PEG 8000 increased the hydrophilic surface and wettability of PRX, resulting in enhancement of diffusion of the dissolution medium and release of PRX.

A $3^{2}$ full factorial design was also used to confirm the effect of temperature and time of the curing process on PRX release from PRX/PEG capsules by curing in a heated coating pan. Results demonstrated irrelevance between the temperature and time of thermal process for curing the physical mixture capsules on the dissolution rate of PRX from the capsules. A temperature of $50-100{ }^{\circ} \mathrm{C}$ for $3-45 \mathrm{~min}$ is sufficient to melt PEG 8000 [15]. After PEG 8000 was melted, the curing process provided the mixing of melted PEG 8000 and PRX. Additionally, lactose and Aerosil ${ }^{\circledR}$ might be dispersed in PRX and PEG 8000 during the curing process.

For the selection of temperature and time of curing process, in which the capsule exhibited the maximum dissolution rate of PRX at $3 \mathrm{~min}$, any temperatures and times could be selected due to the unrelated factors on the dissolution rate of PRX. However, high temperature and long curing time not only provided the completely melted PEG 8000 [14] but also ensured the homogeneous mixing between PRX and PEG 8000 . Therefore, a curing temperature of $70^{\circ} \mathrm{C}$ for $45 \mathrm{~min}$ of physical mixture capsules (F19) was chosen as a candidate to compare with PRX/PEG 8000 melted solid dispersion capsules (F9), free drug capsules (F0) and physical mixture capsules (F10).

The two selected capsule formulations including PRX/PEG melted solid dispersion capsules (F9) and PRX/PEG 8000 physical mixture capsules with curing at $70{ }^{\circ} \mathrm{C}$ for $45 \mathrm{~min}$ (F19) were evaluated for the best formulation, compared to free drug capsules (F0) and PRX/PEG 8000 physical mixture capsules (F10), in which the capsule formulation exhibited the maximum dissolution rate of PRX.

Capsules containing the free drug (F0) provided the slow release which resulted from the poor 
solubility and wettability of PRX, according to the BCS class II of PRX [16]. Capsules containing PRX/PEG 8000 melted solid dispersion (F9), physical mixture (F10) and cured physical mixture (F19) were mainly attributed to increased wettability and improved solubility due to the higher level of hydrophilicity achieved by the use of the polymeric carrier, PEG 8000 [17]. In the dissolution medium, the capsule disintegrated and liberated PRX. PEG 8000 allowed rapid entry of aqueous solutions to PRX.

Moreover, capsules containing PRX/PEG 8000 melted solid dispersion (F9) gave a faster PRX release and dissolution rate, indicating the homogenous solid dispersion between PRX and PEG 8000. The adsorbed PRX on the surface of PEG 8000 was in an extremely fine state, resulting in a decrease in particle size and a concomitant increase in surface area which increased the thermodynamic activity of PRX and enhanced the dissolution of PRX compared to the other formulations [18].

Furthermore, the solid dispersion formulation might create $P R X$ in an amorphous state compared with the physical mixture and free drug capsules, where PRX was presented as a crystalline state [19]. Other mechanisms including the reduction of aggregation and agglomeration of the drug and solubilization of the drug by the carrier of the diffusion layer have been reported to be responsible for improving the aqueous solubility or dissolution properties of solid dispersion formulation [17]. Capsules containing the physical mixture with and without curing process (F19 and F10, respectively) gave the similar dissolution profile and rate. These results indicated that the curing process at $70{ }^{\circ} \mathrm{C}$ for $45 \mathrm{~min}$ of physical mixture capsules did not improve the dissolution of PRX. The PRX was homogeneously dispersed in the PEG 8000 . However, lactose and Aerosi ${ }^{\circledR}$ might disperse in PRX and PEG 8000 during the curing process, causing the interference of hydrophilic surface and wettability of PRX, resulting in slow dissolution rate and hence a similar release of PRX. Results indicated that solid dispersion capsules were the best formula for rapid PRX release. This capsule can be rapidly absorbed to manage painful and inflammatory conditions.

\section{CONCLUSION}

Fast-release PRX/PEG capsules have been successfully prepared by melted solid dispersion, and physical mixture (with and without curing) in a heated coating pan using $3^{2}$ full factorial design. The molecular weight and amount of $P E G$ positively affect the dissolution rate of PRX, whereas curing at different temperatures and duration times do not. Capsules containing PRX/PEG 8000 (1:3 weight ratio) melted solid dispersion exhibit good characteristics with acceptable labeled amount, weight variation and disintegration time. A burst PRX release with rapid dissolution rate is achieved. Therefore, this capsule is a potential immediate release drug delivery system for enhancing the solubility and dissolution rate of low water-soluble drugs.

\section{DECLARATIONS}

\section{Acknowledgement}

The authors would like to acknowledge Faculty of Pharmacy, Rangsit University for laboratory facilities supports. We also thank to Somsuda Tangcham, Siripat Punviriyakool and Pannita Jommalai for their research assistance, and Nawinda Chinatangkul for guidance in DESIGNEXPERT® software.

\section{Conflict of Interest}

No conflict of interest associated with this work.

\section{Contribution of Authors}

The authors declare that this work was done by the authors named in this article and all liabilities pertaining to claims relating to the content of this article will be borne by them.

\section{REFERENCES}

1. Chiou WL, Riegelman S. Increased dissolution rates of water-insoluble cardiac glycosides and steroids via solid dispersions in polyethylene glycol 6000. J Pharm Sci 1971; 65(10): 1569-1571.

2. Afifi S. Solid dispersion approach improving dissolution rate of Stiripentol: a novel antiepileptic drug. Iran $\mathrm{J}$ Pharm Res 2015; 14(4): 1001-1014.

3. Leuner C, Dressman J. Improving drug solubility for oral delivery using solid dispersions. Eur J Pharm Biopharm 2000; 50: 47-60.

4. Yuksel N, Karatas A, Ozkan Y, Savaser A, Ozkan SA, Baykara T. Enhanced bioavailability of piroxicam using Gelucire 44/14 and Labrasol: in vitro and in vivo evaluation. Eur J Pharm Biopharm 2003; 56: 453-459.

5. Prabhu S, Ortega M, Ma C. Novel lipid-based formulations enhancing the in vitro dissolution and permeability characteristics of a poorly water-soluble model drug, piroxicam. Int J Pharm 2005; 301: 209-216.

6. Patel S, Koradia H, Parikh R. Design and development of intranasal in situ gelling system of Midazolam hydrochloride using 32 full factorial design. J Drug Deliv Sci Technol 2015; 30: 154-162. 
7. The United State Pharmacopoeia XXXI/National Formulation XXVI, The United States Pharmacopoeial Convention. Rockville: MD; 2008.

8. Song Y, Wang L, Yang P, Wenslow RM Jr, Tan B, Zhang $H$, Deng Z. Physicochemical characterization of felodipine-kollidon VA64 amorphous solid dispersions prepared by hot-melt extrusion. J Pharm Sci 2013; 102 : 1915-1923.

9. Leu YY, Chow WS. Kinetics of water absorption and thermal properties of poly(lactic acid)/organomontmorillonite/poly(ethylene glycol) nanocomposites. J Vinyl Addit Techn 2011; 17(1): 4047.

10. Vippagunta SR, Wang Z, Hornung S, Krill SL. Factors affecting the formation of eutectic solid dispersions and their dissolution behavior. J Pharm Sci 2007; 96(2): 294-304.

11. Huanga $Y$, Dai WG. Fundamental aspects of solid dispersion technology for poorly soluble drugs. Acta Pharm Sinic B. 2014; 4(1): 18-25.

12. Shah JC, Chen JR, Chow D. Preformulation study of etoposide: II. Increased solubility and dissolution rate by solid-solid dispersions. Int J Pharm 1995; 113: 103-111.

13. Seo A, Holm P, Kristensen HG, Schaefer T. The preparation of agglomerates containing solid dispersions of diazepam by melt agglomeration in a high shear mixer. Int J Pharm 2003; 259: 161-171.

14. Rowe RD, Sheskey PJ, Owen SC. Handbook of pharmaceutical excipients. sixth ed., London: Pharmaceutical Press; 2009. $519 p$.

15. Saharan VA. Current advances in drug delivery through fast dissolving/disintegrating dosage forms: India: Bentham e Books; 2007.

16. Amidon GL, Lennernäs $H$, Shah VP, Crison JR. A theoretical basis for a biopharmaceutic drug classification: The correlation of in vitro drug product dissolution and in vivo bioavailability. Pharm Res 1995; 12: 413-420.

17. Ofokansi KC, Kenechukwu FC, Ezugwu RO, Attama AA. Improved dissolution and anti-inflammatory activity of ibuprofen-polyethylene glycol 8000 solid dispersion systems. Int J Pharm Investig 2016; 6(3): 139-147.

18. Owusu-Ababio G, Ebube NK, Reams R, Habib $M$. Comparative dissolution studies for mefenamic acidpolyethylene glycol solid dispersion systems and tablets. Pharm Dev Technol 1998; 3: 405-412.

19. Guyot M, Fawaz F, Bildet J, Bonini F, Lagueny AM. Physiochemical characterization and dissolution of norfloxacin cyclodextrin complex and PEG solid dispersion. Int J Pharm 1995; 123: 53-63. 